\title{
Spectral autofluorescence imaging of the retina for drusen detection
}

Foubister, James, Gorman, Alistair, Harvey, Andy, Hemert, Jano van

James J. Foubister, Alistair Gorman, Andy Harvey, Jano van Hemert, "Spectral autofluorescence imaging of the retina for drusen detection," Proc. SPIE 10474, Ophthalmic Technologies XXVIII, 104741H (19 February 2018); doi: $10.1117 / 12.2286611$

SPIE. Event: SPIE BiOS, 2018, San Francisco, California, United States 


\title{
Spectral Autofluorescence imaging of the retina for drusen detection
}

\author{
James J. Foubister ${ }^{\mathrm{a}, \mathrm{b}}$, Alistair Gorman ${ }^{\mathrm{a}}$, Andy Harvey ${ }^{\mathrm{b}}$, and Jano van Hemert ${ }^{\mathrm{a}}$ \\ a Optos Plc, Queensferry House, Carnegie Campus, Dunfermline, United Kingdom \\ bImaging Concepts Group, School of Physics and Astronomy, Kelvin Building, University of \\ Glasgow, Glasgow, United Kingdom
}

\begin{abstract}
The presence and characteristics of drusen in retinal images, namely their size, location, and distribution, can be used to aid in the diagnosis and monitoring of Age Related Macular Degeneration (AMD); one of the leading causes for blindness in the elderly population. Current imaging techniques are effective at determining the presence and number of drusen, but fail when it comes to classifying their size and form. These distinctions are important for correctly characterising the disease, especially in the early stages where the development of just one larger drusen can indicate progression. Another challenge for automated detection is in distinguishing them from other retinal features, such as cotton wool spots.

We describe the development of a multi-spectral scanning-laser ophthalmoscope that records images of retinal autofluorescence (AF) in four spectral bands. This will offer the potential to detect drusen with improved contrast based on spectral discrimination for automated classification. The resulting improved specificity and sensitivity for their detection offers more reliable characterisation of AMD. We present proof of principle images prior to further system optimisation and clinical trials for assessment of enhanced detection of drusen.
\end{abstract}

Keywords: Retinal imaging, Scanning laser ophthalmoscope, Drusen, Age-related macular degeneration, Autofluorescence, Spectral imaging

\section{INTRODUCTION}

One of the most significant causes of vision loss worldwide, and the main cause for blindness in developed countries, is Age-Related Macular Degeneration (AMD). ${ }^{1,2}$ The disease is most common in those over 50, and the risk factors increase as age progresses. It is currently predicted that the number of people suffering from the condition is likely to increase 1.5 times in the next 10 years. ${ }^{1}$ If action is not taken, then the issue is only going to compound, creating a larger burden on countries and health-care providers. ${ }^{3}$

Fortunately as AMD is one of the most prevalent ocular diseases, along with the likes of Glaucoma and Diabetic Retinopathy, understanding of its progression and symptoms are well explored. The current preferred practice patterns published by the American Academy of Ophthalmology break the disease down into a number of significant stages, namely Early, Intermediate, and Advanced AMD. ${ }^{4}$ These stages are most commonly diagnosed and defined by the characterisation of drusen, small, yellow, exudative deposits that form between the Bruch's membrane and the retinal pigment epithelium of the retina. ${ }^{5}$ They can and often do occur naturally in anyone above the age of 40, and are non-harmful in isolation. ${ }^{5}$ However, the largest risk factor for the progression of the different stages of AMD are the characteristics of the drusen, namely their number, distribution, and most importantly, their size. ${ }^{6}$ Therefore by monitoring the drusen present on the retina, AMD can be diagnosed and characterised.

At the early stages of AMD, clinicians would expect to see a number of smaller hard drusen $(<63 \mu \mathrm{m})$, with a few intermediate drusen $(63-124 \mu \mathrm{m})$. Intermediate AMD is a more significant diagnosis as approximately $18 \%$ of this group will progress to further stages of the disease within 5 years. ${ }^{7}$ It is characterised with the presence of a larger number of intermediate drusen, along with at least one large soft drusen $(>125 \mu \mathrm{m})$. Advanced AMD

Further author information: (Send correspondence to J.J.F.)

J.J.F.: E-mail: jf88@hw.ac.uk

Ophthalmic Technologies XXVIII, edited by Fabrice Manns, Per G. Söderberg, Arthur Ho, Proc. of SPIE

Vol. 10474, 104741H · @ 2018 SPIE · CCC code: 1605-7422/18/\$18 · doi: 10.1117/12.2286611

Proc. of SPIE Vol. $10474104741 \mathrm{H}-1$ 
will see further progression of these drusen, but also much more serious symptoms with the development of either geographic atrophy, a permanent death of retinal cells, or Neovascular AMD, the growth of abnormal blood vessels which damage the macula. These two developments can also be referred to as Dry AMD and Wet AMD respectively. ${ }^{7}$ Fig. 1 shows an image of a retina, recorded using a conventional fundus camera, containing a significant number of larger drusen.

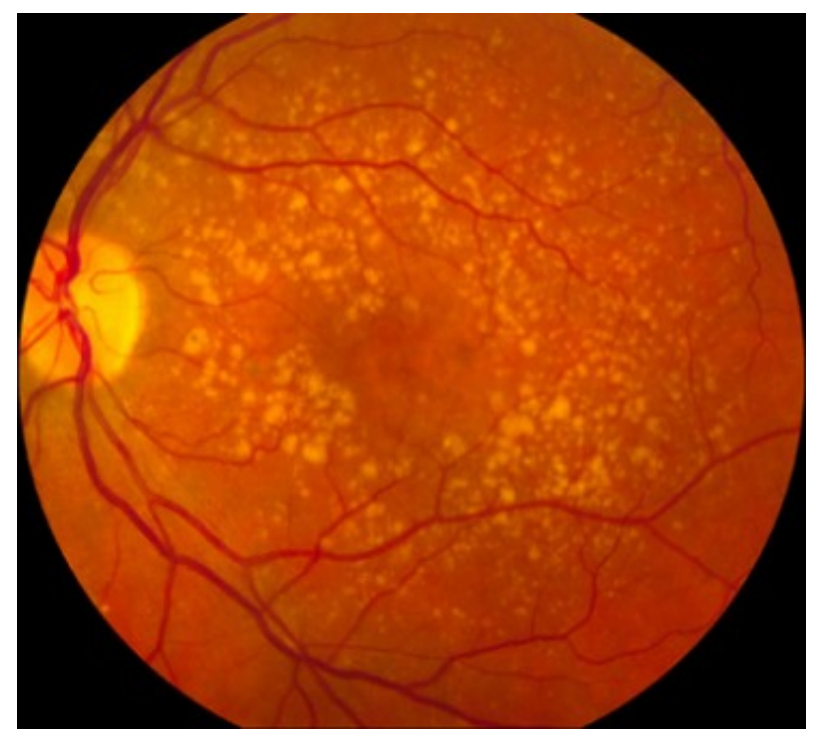

Figure 1. Fundus reflectance image of a retina which contains a large number of large soft drusen. ${ }^{8}$

Unfortunately there is no cure for AMD, however there are treatment options that have been shown to slow the progression of the disease, ${ }^{7,9}$ with the treatment varying depending on the diseases stage. Dietary and nutrition plans are attempted at the earlier stages with the aim of trying to reduce the risk factors such as smoking and obesity. ${ }^{9}$ While at the later stages, such as Wet AMD, laser surgery can be employed to aid in preventing further vision loss. ${ }^{10}$ Clearly it is beneficial to halt or delay the diseases progression at the earlier stages. Therefore it is vitally important that any detection and characterisation method can provide clinicians the certainty they require to diagnose at the earliest possible opportunity.

The most common current diagnostic techniques vary from standard fundus photography ${ }^{11}$ and OCT, ${ }^{12}$ to the more invasive method of fluorescein angiography. ${ }^{13}$ The main issues with these techniques is that while they are effective at detecting the presence of drusen, they fail when it comes to classifying whether they are hard (small) or soft (large). ${ }^{1}$ This distinction is vitally important for AMD classification, especially at the early stages, and is why current techniques cannot be relied upon, as it is the development of larger drusen that herald the diseases progression. ${ }^{4,7}$

The other major issues with these techniques are the lack of reliability. Many of these techniques require a time consuming manual grading process, which can lead to human mistake and error. ${ }^{14}$ Others utilise automated detection methods, which too have proved ineffective at classifying the different forms of Drusen. ${ }^{15}$ Therefore, to aid in diagnosing this disease at early stages, a technique is required which can not only detect the presence of drusen, but also accurately determine their form and size. This process should also not have to rely on the inconsistent grading methods of the previous techniques. Producing a technique which can solve these issues is the aim of this project. A new practical in eye method for detecting drusen will be produced, which can provide the information necessary for clinicians to diagnose AMD to the high level of certainty required.

\section{SPECTRAL AUTOFLUORESCENCE TECHNIQUE}

To accomplish this aim, an innovative technique that has been demonstrated by a multi-institution group based out of New York University is being adapted. Titled, "Hyperspectral Autofluorescence Imaging of Drusen and 
Retinal Pigments Epithelium in Donor Eyes with Age-Related Macular Degeneration", the aim of this research was to identify the spectral autofluorescence characteristics of drusen and the retinal pigment epithelium, from eyes which had been suffering from AMD. ${ }^{16}$

As eluded to in the title, the group carried out a simple hyperspectral autofluorescence investigation on flat mounted retinal samples. The flat mounted samples are sections of the retina which have had the outer ocular media (i.e. the lens, cornea) removed, as well as some retinal layers, such as the sclera. ${ }^{17}$ Removal of these layers provides a relatively unobstructed signal of the retina for the their process to investigate. This means that interfering effects, such as absorption or AF emission from these layers do not have to be considered, making it easier for the remaining layers spectra to be extracted. Exciting the retina at $436 \mathrm{~nm}$ and $480 \mathrm{~nm}$, spectral AF data was gathered in a wavelength range of $480 \mathrm{~nm}-720 \mathrm{~nm}$, the group then analysed the data with a machinelearning non-negative tensor-factorisation algorithm. ${ }^{18}$ This produced a number of spatially localised spectra which could be directly attributed to different layers or features in the retina, one of them specifically being drusen.

This information allows the technique to produce images of their retinal samples in which the drusen are highlighted brightly, clearly standing out over the background noise of the rest of the image. This is exactly the kind of process that is needed by clinicians to identify drusen at the early stages of AMD. Even more so, if the drusen can be highlighted as clearly as they have been in this technique, then there is the potential that automatic grading methods can be used. ${ }^{19}$ This would remove the need for the manual grading process which causes significant uncertainty and error in current imaging techniques.

The major issue with the current technique is that it has only been demonstrated on extracted retinal samples, and not in vivo. As mentioned, this means that the spectral signals being analysed are more ideal than they would be in eye imaging. In vivo this technique would have to take into account additional autofluorescence signals from these components, such as the ocular lens, ${ }^{20}$ as well as the basic losses incurred from absorption or scattering in the outer ocular media. ${ }^{21}$ It would also have additional restrictions due to optical eye safety rules which are particularly limiting at the low wavelengths used $(436 \mathrm{~nm}, 480 \mathrm{~nm}) .{ }^{22}$

The information and methods divulged in this technique are significant. If implemented successfully in a retinal imaging device, it would be a particularly useful tool for the characterisation and detection of AMD. While there are significant challenges to accomplishing this, it is for this reason that this technique has been chosen. Utilising the information presented, a retinal in vivo system will be adapted and constructed, before being tested on patients in small scale clinical trials. The intention of the trials would be to investigate the techniques specificity and sensitivity for detecting drusen when compared alongside a more traditional fundus imaging technique.

\section{METHOD}

With a full understanding both of the main function of the Spectral AF technique, and an awareness of its current limitations, the task of converting it to work within a human eye is a significant one. What follows is the original intended design of a multi-Spectral AF device which would be capable of performing the technique within the human eye. A simplified version of the devices optical layout can be seen in Fig. 2.

Our multi-spectral AF scanning laser ophthalmoscope employs a conventional raster-scan, enhanced by two excitation sources, $450 \mathrm{~nm}$ and $488 \mathrm{~nm}$. The excitation sources are fibre coupled into the AF SLO using a dichroic beam splitter that reflects the shorter-wavelength excitation light and transmits the longer-wavelength fluorescence. The excitation light is collimated and transmitted to the scanners, which consists of a slow vertical galvo scanner, and a fast horizontal resonant scanner. This raster output reflects off a cold mirror, passing into the simple dual lens barrel. The lens barrel directs the beam, ensuring it comes to a focal point in the centre of the patients pupil, before scanning across the back of the retina. In this configuration a field of view of $40^{\circ}$ internal angle is achievable. For context, the back hemisphere inside the eyeball where the retina sits would be considered a $180^{\circ}$ internal angle. Ideally a larger area of the retina would be imaged, to identify as many drusen as possible, however the angle is currently dictated by the system set up. Expanding this technique to work in wide field systems could be a part of future work. 


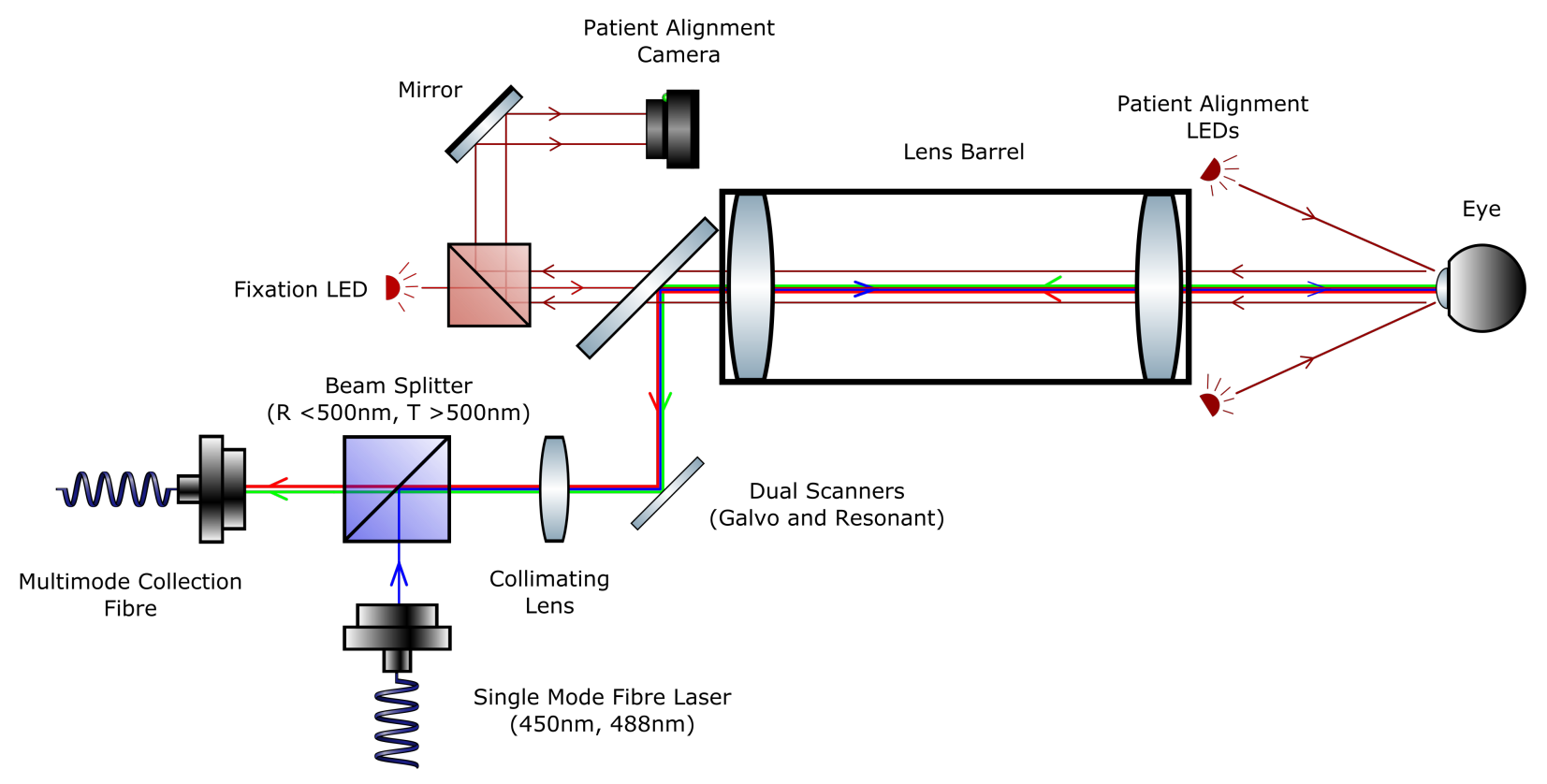

Figure 2. Simplified optical layout of multi-spectral AF device for in vivo imaging. Detection components omitted.

As the excitation beam scans across the retina it excites fluorophores contained within the different layers. These emit, with their wavelength shifted fluorescence emitting back out of the pupil, and through the system. The signal is de-scanned by the scanning mirrors, focused, transmitted through the dichroic beam splitter, and collected in a multimode fibre. The size of the multimode fibre core determines the systems confocality, as well as how much of the AF signal is captured. For a high SNR a large fibre would be used, however the contrast and resolution of the images decreases, with the inverse being the case for a small fibre.

Once collected, the fluorescence signal can be interrogated to identify drusen present in the images. In an ideal system, the retinal AF signal would be analysed with a hyperspectral method, as demonstrated in the original technique, ${ }^{16}$ being split across hundreds of spectral bands. Due to the reduced signal strength available in in vivo imaging this is not achievable, and the method must be converted to multispectral, utilising a much smaller number of bands to sacrifice spectral resolution for increased signal strength. This convention of switching from hyperspectral to multispectral is very common in new techniques once the investigation process is complete, mainly to reduce cost and increase SNR.

For this method, 4 spectral bands have been identified, ranging from $500 \mathrm{~nm}-700 \mathrm{~nm}$. Once collected, the fluorescence signal is separated into these 4 bands using dichroic beam splitters. The individual signals are then simultaneously captured by Avalanche photo diodes. These bands have been specifically chosen so that the base spectra identified in the original technique ${ }^{16}$ can be separated, both spectrally and spatially. For example, band 1 has drusen fluorescing strongly, while in band 2 the signal has dropped. Over both these bands, the spectra most associated with the RPE fluoresces strongly. Therefore by taking a simple difference calculation, the two bands can be separated, spatially and spectrally. This method is repeated for the other notable spectra and will allow the drusen spectra to be isolated above the background retinal noise, producing images where the drusen can be easily spatially located.

Also included in the system are a number of features to aid in the alignment of patients, to help improve image quality and SNR. These consist of a simple fixation LED, providing a target for the patient to fixate on. As well as a trio of IR Patient Alignment LEDs, which reflect off the patients cornea, and back to a camera, providing the operator with information with which they can align the device. 


\section{PRACTICAL PROGRESS}

\subsection{Optics}

The current device is an older Infra-red Optical coherence tomography system that has undergone heavy modification. The optical path has been changed significantly and now resembles the layout detailed in Fig. 2. The device is functional, with retinal reflectance images being captured at a number of visible wavelengths $(450 \mathrm{~nm}$, $532 \mathrm{~nm}, 635 \mathrm{~nm}$ ), see Fig. 3. Preliminary retinal autofluorescence images have also been obtained. The produced images are in focus, with a sufficient resolution to see drusen, at around $30 \mu \mathrm{m}$. The signal to noise ratio of these images however is still lacking. SNR is always going to be a major challenge for any retinal AF system and a number of approaches are available to tackle this. These include increasing the size of the core of the multi-mode collection fibre to gather additional signal, and using mydriasis to increase the pupil size of the patient, increasing out limiting aperture and allowing more of the retinal AF signal to be captured by the system.
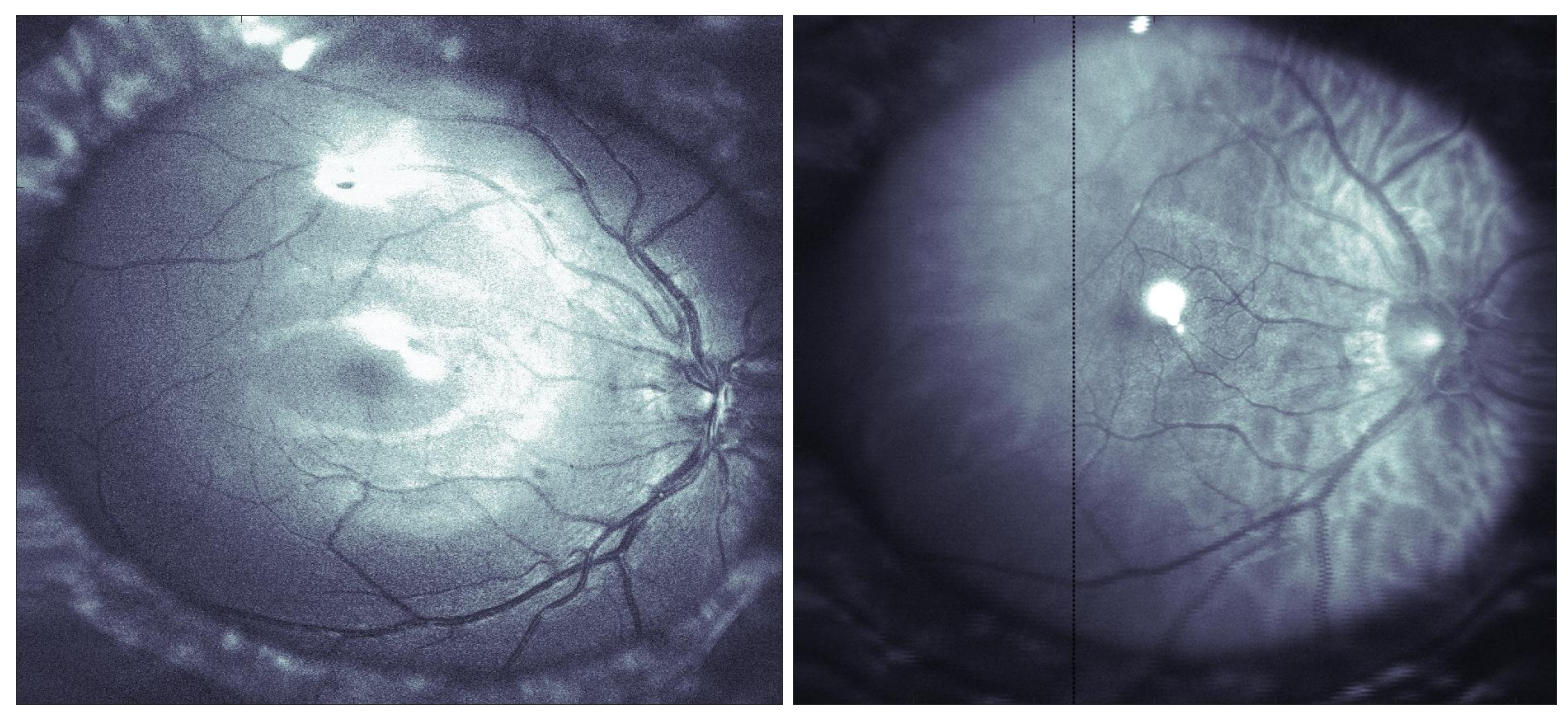

Figure 3. $450 \mathrm{~nm}$ and $633 \mathrm{~nm}$ reflectance images captured with system.

\subsection{Maximum Permissible Exposure}

For retinal imaging, there are a series of rules which dictate the safe light-irradiation levels permitted. Referred to as the Maximum Permissible Exposure limits (MPE), ${ }^{22}$ these rules take into account wavelength, exposure duration, and area of retinal exposure. The formulas provided can then be used to calculate the safe optical power limits that guarantee no risk of retinal damage.

Two main forms of optical damage are considered: thermal and photochemical. The thermal damage calculations model the effects of heat build-up and dissipation over the retina. This effect occurs at all wavelengths, but is generally less severe towards the IR end of the spectrum. Photochemical damage can occur when light is absorbed by a chromophore on the retina, exciting an electron from its ground state. This can split bonds and generate free radicals which attack and damage retinal tissue. ${ }^{23,24}$ It occurs at short wavelengths (400nm$600 \mathrm{~nm})$ and, unlike thermal damage, is an additive effect. Photons of the specified wavelengths work to break these bonds, with it taking a certain specified amount to do so. This make the effect entirely independent of exposure duration, instead purely driven by total energy transferred. The additive effects need to be considered over a time period of 24 hours as after this duration repair mechanisms reverse the damage done. ${ }^{24}$ A comparison of the two limits for a stationary point laser source on the retina can be seen in Fig. 4.

Due to the short scanning durations of the device, the thermal effect has not been an issue, and the main challenge has arisen in dealing with the photochemical effect. The excitation sources used (450nm, 488nm) lie at the more harmful and restrictive ends of the effect. Furthermore, the additive characteristic directly limits the amount of retinal exposures that can be carried out in a 24 hour period. ${ }^{24}$ 


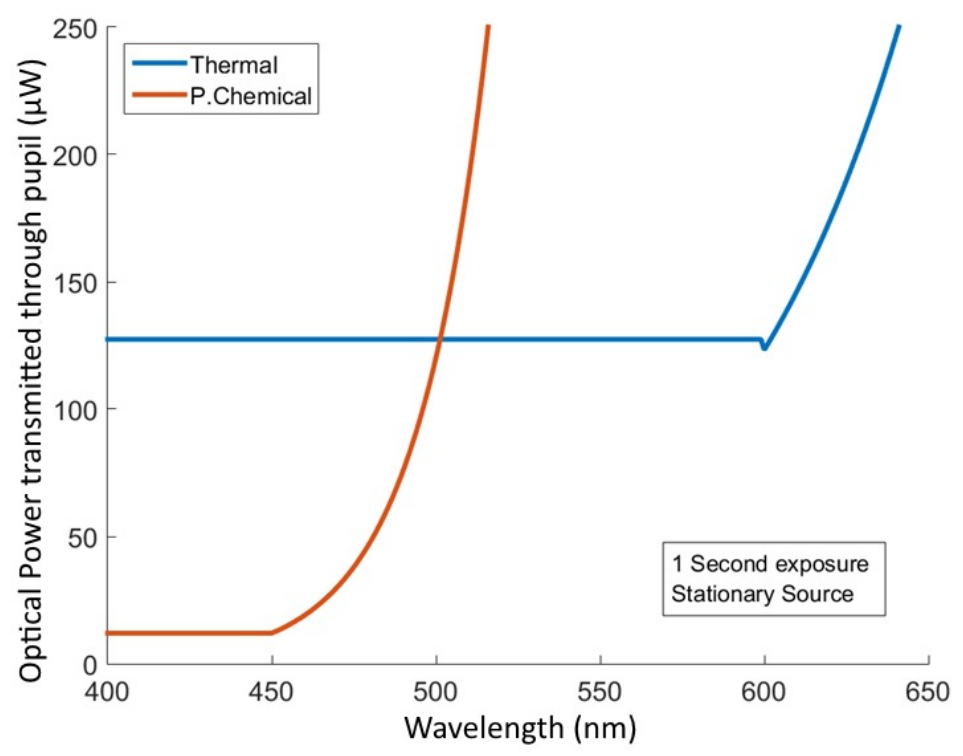

Figure 4. Thermal and Photochemical limitations for a laser focused onto the retina. Total continuous optical power in $\mu \mathrm{W}$ permitted to transmit through the pupil for 1 second.

Both effects are more restrictive when a laser beam is stationary on the retina. However, if that beam can be scanned very quickly, then it emulates a continuous wave beam uniformly distributed over the exposed area. ${ }^{22}$ While not reducing the immediate peak power, fast scanning does greatly reduce the averaged accumulated power and this allows the photochemical restrictions to be somewhat relaxed. To accomplish this a $4 \mathrm{kHz}$ resonant scanner has been employed into the system to act as the horizontal fast scan. This allows full, high resolution images to be captured in under 0.5 seconds, and also significantly reduces the risk and limitation of photochemical effect.

\subsection{Safety Circuit}

Due to these harsh limitations on the permissible exposure, the risk of damage to the patient should the device malfunction is significant. It therefore must be ensured that a reliable safety circuit is in place to guarantee that should any of the devices failure modes occur, it can shut down in a safe and prompt fashion. The main failure modes of the device are increases in laser power, and a malfunction of the scanning configuration.

Confirming that the lasers remain at a steady power during imaging is a straightforward procedure. A 10:90 beam splitter is placed in the optics path, skimming a representative $10 \%$ of the incident power before it enters the system. These values are closely monitored using silicon photo detectors and simple single-board computers to guarantee that should the laser power change abnormally, dropping to high or low, then the scanning system will be shut down safely.

The operation of the resonant scanner is monitored using simple microphones located near the scanner to record the associated $4 \mathrm{kHz}$ acoustic tone. A simple algorithm monitors the sinusoidal tone and the devices external cladding protects the microphone from background noise. Once a failure signal has been detected, the system follows a safe shut-down protocol immediately to remove any risk to the patient. The full logic safety circuit of the device can be seen in Fig. 5

\section{CONCLUSIONS}

At this stage of the project, a number of things have been achieved. Most importantly our multispectral AF technique has been decided upon. Utilising the research carried out in the original spectral autofluorescence technique ${ }^{16}$ we have decided on 4 multispectral bands that will allow us to detect drusen on the retina in vivo. A device which can accomplish this has been constructed, and has been shown to be capable of capturing retinal 


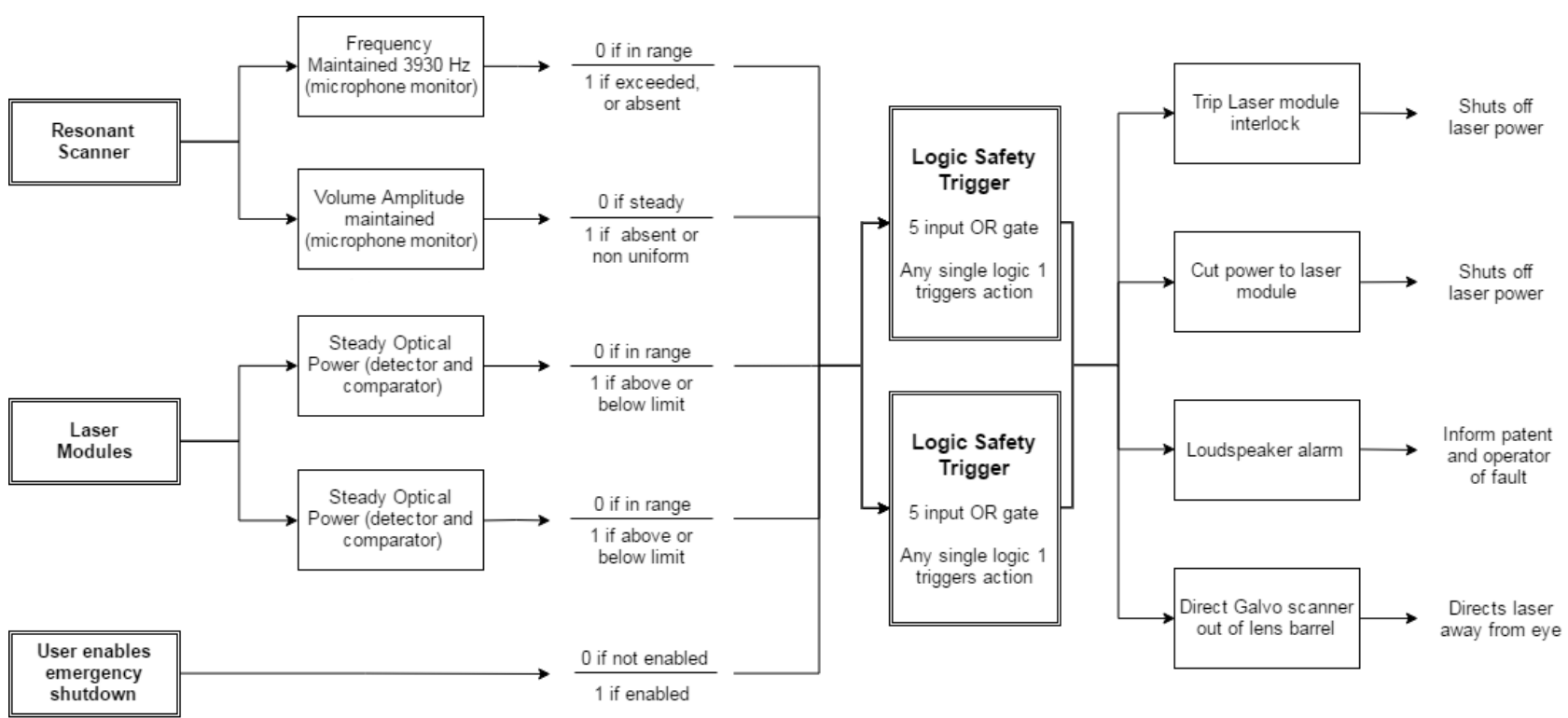

Figure 5. Safety Circuit logic diagram. Capable of detecting failure modes of the Scanning configuration, and the laser modules.

reflectance, and preliminary autofluorescence images. Current developmental focus is on increasing the signal quality of these images. The necessary support modules, such as the safety circuit, are in place, and the device stands ready to move towards the testing stages.

To investigate the effectiveness of the technique, the device will be placed into two small scale clinical trials. The first of these will run in house at Optos Plc, and will image volunteers. The likelihood of detecting drusen in this trial will be low as there will be no opportunity for pre selection of the patients. It will however act as an intensive test run for the device as well as providing a bank of healthy eye data. The second trial will be held at the University of Glasgow and, with the aid of clinical partners, image AMD affected patients. This should provide retinas which contain a wide variety and number of drusen, and also patients who are elderly and therefore similar to the target demographic. Ideally our technique will be placed along side a more traditional fundus imaging technique, which will allow full characterisation of the techniques specificity and sensitivity for detecting drusen and diagnosing AMD.

Some major challenges still lie ahead. Perhaps the most significant of these are the SNR limitations that stem from the exposure limits. There are a number of possible solutions that are being explored. The first of these has been discussed and is the inclusion of the fast resonant scanner. The device being treated as a uniform distribution has allowed a more intense beam to be used in the imaging process. Another option is increasing the size of the collection fibre core. As long as the tissue point spread function is sufficiently large more retinal signal can be captured. However, increasing the diameter of the fibre results in a direct trade off between SNR and image contrast. The most ideal balance will be found with a short investigation into different fibre cores.

The use of mydriasis would be another option that provides scope to increase the sensitivity of the device by an order of magnitude. Increasing the diameter of the patients pupil will allow more AF signal to escape the eye, resulting in more signal to be captured at the collection fibre. This does have limitations though, in that the techniques usability in some clinical settings will be restricted. It makes the technique significantly more invasive, for example it cannot be used on patients who are short sighted for the risk of causing acute angle closure glaucoma. ${ }^{25}$ It therefore does not want to be relied upon as a necessary method, however for the clinical trials it can still be useful to establish the potential of the technique if the signal cannot be increase through other means.

To conclude, the goal of this project is to provide a technique that can detect and classify drusen, providing clinicians the certainty they require to diagnose AMD at the earliest stages. To accomplish this a multispectral 
AF technique has been established and an appropriate device constructed. Retinal imaging has been carried out in vivo, obtaining reflectance and autofluorescence images. The technique is now quickly moving towards the clinical testing stages where it will be compared alongside traditional drusen detection techniques, establishing their ability to classify drusen. A number of challenges remain, but with sufficient foresight and preparation, should not impede progress.

\section{REFERENCES}

[1] Kanagasingam, Y., Bhuiyan, A., Abramoff, M. D., Smith, R. T., Goldschmidt, L., and Wong, T. Y., "Progress on retinal image analysis for age related macular degeneration," SURVEY OF OPHTHALMOLOGY 38, 20-42 (2014).

[2] Congdon, N., O’Colmain, B., Klaver, C., Klein, R., Munoz, B., Friedman, D., Kempen, J., Taylor, H., Mitchell, P., Hyman, L., and Grp, E. D. P. R., "Causes and prevalence of visual impairment among adults in the united states," ARCHIVES OF OPHTHALMOLOGY 122(4), 477-485 (2004).

[3] [The State of the Nation; Eye Health 2016], Royal National Institute of Blind People (2016).

[4] American Academy of Ophthalmology, "Age-Related Macular Degeneration PPP - Updated 2015." https: //www.aao.org/preferred-practice-pattern/age-related-macular-degeneration-ppp-2015 (2015).

[5] Williams, M. A., Craig, D., Passmore, P., and Silvestri, G., "Retinal drusen: harbingers of age, safe havens for trouble," AGE AND AGEING 38(6), 648-654 (2009).

[6] Pauleikhoff, D., Barondes, M., Minassian, D., Chisholm, I., and Bird, A., "Drusen as risk-factors in agerelated macular disease," AMERICAN JOURNAL OF OPHTHALMOLOGY 109(1), 38-43 (1990).

[7] National Eye Institute, "Facts About Age-Related Macular Degeneration." https://nei.nih.gov/health/ maculardegen/armd_facts (2015).

[8] National Eye Institute, "NEI Photos and Images: Age-Related Macular Degeneration." https://nei.nih. gov/photo/amd.

[9] Meyers, K. J., Liu, Z., Millen, A. E., Iyengar, S. K., Blodi, B. A., Johnson, E., Snodderly, D. M., Klein, M. L., Gehrs, K. M., Tinker, L., Sarto, G. E., Robinson, J., Wallace, R. B., and Mares, J. A., "Joint associations of diet, lifestyle, and genes with age-related macular degeneration," OPHTHALMOLOGY 122(11), 2286-2294 (2015).

[10] Yonekawa, Y., Miller, J. W., and Kim, I. K., "Age-related macular degeneration: Advances in management and diagnosis," JOURNAL OF CLINICAL MEDICINE 4(2), 343-359 (2015).

[11] Bartlett, H. and Epejesi, F., "Diagnostic and surgical techniques - use of fundus imaging in quantification of age-related macular change," SURVEY OF OPHTHALMOLOGY 52(6), 655-671 (2007).

[12] Schlanitz, F. G., Ahlers, C., Sacu, S., Schuetze, C., Rodriguez, M., Schriefl, S., Golbaz, I., Spalek, T., Stock, G., and Schmidt-Erfurth, U., "Performance of drusen detection by spectral-domain optical coherence tomography," INVESTIGATIVE OPHTHALMOLOGY \& VISUAL SCIENCE 51(12), 6715-6721 (2010).

[13] Friedman, D., Parker, J. S., Kimble, J. A., Delori, F. C., McGwin, Jr., G., and Curcio, C. A., "Quantification of fluorescein-stained drusen associated with age-related macular degeneration," RETINA 32(1), 19-24 (2012).

[14] Bhuiyan, A., Kawasaki, R., Sasaki, M., Lamoureux, E., and Ramamohanarao, K., "Drusen detection and quantification for early identification of age related macular degeneration using color fundus imaging," Journal of Clinical and Experimental Ophthalmology 4(5) (2013).

[15] Bhuiyan, A., Xiao, D., and Kanagasingam, Y., "A review of disease grading and remote diagnosis for sight threatening eye condition: Age related macular degeneration," Journal of Computer Science $\&$ System Biology 7, 62-071 (2014).

[16] Tong, Y., Ami, T. B., Hong, S., Heintzmann, R., Gerig, G., Ablonczy, Z., Curcio, C. A., Ach, T., and Smith, R. T., "Hyperspectral autofluorescence imaging of drusen and retinal pigments epithelium in donor eyes with age-related macular degeneration," Retina 36, S127-S136 (2016).

[17] Ach, T., Huisingh, C., McGwin, Jr, G., Messinger, J. D., Zhang, T., Bentley, M. J., Gutierrez, D. B., Ablonczy, Z., Smith, R. T., Sloan, K. R., and Curcio, C. A., "Quantitative autofluorescence and cell density maps of the human retinal pigment epithelium," Invest Ophthalmol Vis Sci 55(8), 4832-4841 (2014). 
[18] Smith, R., Post, R., Johri, A., Lee, M., Ablonczy, Z., Curcio, C., Ach, T., and Sajda, P., "Simultaneous decomposition of multiple hyperspectral data sets: signal recovery of unknown fluorophores in the retinal pigment epithelium," Biomedical Optics Express 5(12), 4171-4185 (2014).

[19] van Grinsven, M. J. J. P., Lechanteur, Y. T. E., van de Ven, J. P. H., van Ginneken, B., Hoyng, C. B., Theelen, T., and Sanchez, C. I., "Automatic drusen quantification and risk assessment of age-related macular degeneration on color fundus images," Invest Ophthalmol Vis Sci 54(4), 3019-3027 (2013).

[20] SATOH, K., BANDO, M., and NAKAJIMA, A., "Fluorescence in human lens," EXPERIMENTAL EYE RESEARCH 16(2), 167-172 (1973).

[21] MELLERIO, J., "Light absorption and scatter in human lens," VISION RESEARCH 11(2), 129-141 (1971).

[22] Delori, F. C., Webb, R. H., and Sliney, D. H., "Maximum permissible exposures for ocular safety (ansi 2000), with emphasis on ophthalmic devices," JOURNAL OF THE OPTICAL SOCIETY OF AMERICA A-OPTICS IMAGE SCIENCE AND VISION 24(5), 1250-1265 (2007).

[23] Youssef, P. N., Sheibani, N., and Albert, D. M., "Retinal light toxicity," EYE 25(1), 1-14 (2011).

[24] Wu, J., Seregard, S., and Algvere, P. V., "Photochemical damage of the retina," Survey of Ophthalmology 51(5), 461-481 (2006).

[25] Lachkar, Y. and Bouassida, W., "Drug-induced acute angle closure glaucoma," CURRENT OPINION IN OPHTHALMOLOGY 18(2) (2007). 